\title{
Power and Performance Evaluation of Memcached on the TILEPro64 Architecture
}

\author{
Mateusz Berezecki \\ Facebook \\ mateuszb@fb.com
}

\author{
Eitan Frachtenberg \\ Facebook \\ etc@fb.com
}

\author{
Mike Paleczny \\ Facebook \\ mpal@fb.com
}

\author{
Kenneth Steele \\ Tilera \\ ken@tilera.com
}

October 10, 2011

\begin{abstract}
Power consumption of data centers had become an important factor in the economy and sustainability of large-scale Web services. Researchers and practitioners are spending considerable effort to characterize Web-scale workloads and evaluating their applicability to alternative, more power-efficient architectures. One such workload in particular is the caching layer, which stores expensive-to-regenerate data in fast storage to reduce service times. In this paper we look at one such application, the Memcached key-value store, which is widely deployed at Facebook and other Web services, and one alternative architecture, the TILEPro64 many-core system-on-chip. We explore the performance and power characteristics of Memcached under a variety of workload variations, implementation choices, and communication protocol, and compare them to a traditional implementation on $\mathrm{x} 86$-based servers. Our results suggest that the TILEPro64 architecture can significantly outperform x86-based architectures in terms of throughput per Watt for the evaluated version of Memcached.
\end{abstract}

Keywords: tilera; memcached; low power; many-core processors; key-value store

\section{Introduction}

Key-value (KV) stores play an important role in many large websites. Examples include: Dynamo at Amazon [1]; Redis at Github, and Blizzard Interactive ${ }^{1}$; Memcached at Facebook, Zynga and Twitter [2,3]; and Voldemort at Linkedin ${ }^{2}$.

\footnotetext{
${ }^{1}$ http://redis.io

${ }^{2}$ http://project-voldemort.com
} 
All these systems store ordered (key,value) pairs and are, in essence, a distributed hash table.

A common use case for these systems is as a layer in the data-retrieval hierarchy: a cache for expensive-to-obtain values, indexed by unique keys. These values can represent any data that is cheaper or faster to cache than re-obtain, such as commonly accessed results of database queries or the results of complex computations that require temporary storage and distribution.

Because of their role in data-retrieval performance, $\mathrm{KV}$ stores attempt to keep much of the data in main memory, to avoid expensive I/O operations $[4,5]$. Some systems, such as Redis or Memcached, keep data exclusively in main memory. In addition, KV stores are generally network-enabled, permitting the sharing of information across the machine boundary and offering the functionality of large-scale distributed shared memory without the need for specialized hardware.

This sharing aspect is critical for large-scale web sites, where the sheer data size and number of queries on it far exceed the capacity of any single server. Such large-data workloads can be I/O intensive and have no obvious access patterns that would foster prefetching. Caching and sharing the data among many frontend servers allows system architects to plan for simple, linear scaling, adding more KV stores to the cluster as the data grows. At Facebook, we have used this property grow larger and larger clusters, and scaled Memcached accordingly ${ }^{3}$.

But as these clusters grow larger, their associated operating cost grows commensurably. The largest component of this cost, electricity, stems from the need to power more processors, RAM, disks, etc. Lang [6] and Andersen [4] place the cost of powering servers in the data center at up to $50 \%$ of the three-year total ownership cost (TCO). Even at lower rates, this cost component is substantial, especially as data centers grow larger and larger every year [7].

One of the proposed solutions to this mounting cost is the use of so-called "wimpy" nodes with low-power CPUs to power KV stores [4]. Although these processors, with their relatively slow clock speeds, are inappropriate for many demanding workloads [6], KV stores can present a cost-effective exception because even a slow CPU can provide adequate performance for the typical $\mathrm{KV}$ operations, especially when including network latency.

In this paper, we focus on a different architecture: the Tilera TILEPro64 64-core CPU $[8,9,10,11,12]$. This architecture is interesting for a Memcached workload in particular (and KV stores in general), because it combines the low-power consumption of slower clock speeds with the increased throughput of many independent cores (described in detail in Sections 2 and 3). As mentioned above, previous work has mostly concentrated on mapping KV stores to lowcore-count "wimpy" nodes (such as the Intel Atom), trading off low aggregate power consumption for a larger total node count [4]. This trade-off can mean higher costs for hardware, system administration, and fault management of very large clusters.

\footnotetext{
${ }^{3}$ For example, see facebook. com/note.php?note_id=39391378919 for a discussion of Facebook's scale and performance issues with Memcached.
} 
Our initial evaluation of the TILEPro64 architecture [13] found encouraging efficiency advantages on a limited set of workloads, compared to x86-based systems. In this work, we expand upon our previous results in three areas:

1. We enhance the workload to include not only read operations but also various mixes of reads and writes, and their effect on the combined maximum throughput attainable.

2. We enhance the usage model to include not only the UDP protocol for reads, but also TCP (writes always use TCP). The choice of protocol affects not both the latency and throughput of read requests, and to a lesser degree, the power consumption of the node.

3. We further tuned our stock version of Memcached to eliminate some of the locking bottlenecks we previously identified, thus improving the overall throughput on the x86-based systems.

\section{Memcached Architecture}

Memcached ${ }^{4}$ is a simple, open-source software package that exposes data in RAM to clients over the network. As data size grows in the application, more RAM can be added to a server, or more servers can be added to the network. In the latter case, servers do not communicate among themselves - only clients communicate with servers. Clients use consistent hashing [14] to select a unique server per key, requiring only the knowledge of the total number of servers and their IP addresses. This technique presents the entire aggregate data in the servers as a unified distributed hash table, keeps servers independent, and facilitates scaling as data size grows.

Memcached's interface provides all the basic primitives that hash tables provide - insertion, deletion, and lookup/retrieval - as well as more complex operations built atop them. The complete interface includes the following operations:

- STORE: stores (key,value) in the table.

- ADD: adds (key, value) to the table iff the lookup for key fails.

- DELETE: deletes (key,value) from the table based on key.

- REPLACE: replaces $\left(k e y, v_{a l u e_{1}}\right)$ with $\left(k e y, v_{a l u e_{2}}\right)$ based on $\left(k e y, v_{a l u e_{2}}\right)$.

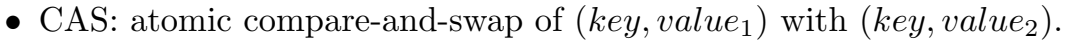

- GET: retrieves either (key,value) or a set of $\left(k_{e} y_{i}, v_{a l u e}\right)$ pairs based on $k e y$ or $\left\{k e y_{i}\right.$ s.t. $\left.i=1 \ldots k\right\}$.

\footnotetext{
${ }^{4}$ http://memcached.org/
} 


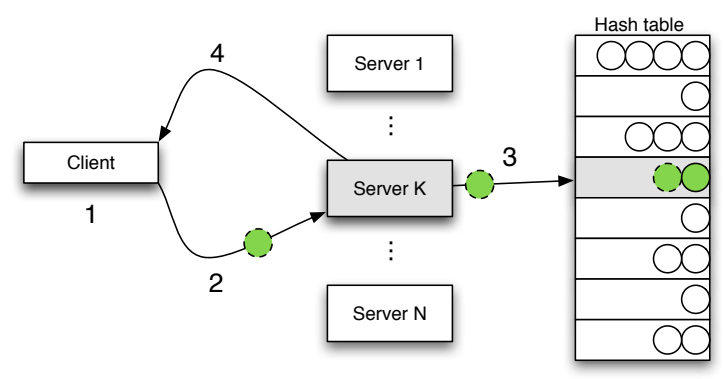

Figure 1: Write path: The client selects a server (1) by computing $k_{1}=$ consistent_hash $_{1}($ key $)$ and sends (2) it the $(k e y, v a l u e)$ pair. The server then calculates $k_{2}=h_{a s h}(k e y) \bmod M$ using a different hash function and stores (3) the entire (key,value) pair in the appropriate slot $k_{2}$ in the hash table, using chaining in case of conflicts. Finally, the server acknowledges (4) the operation to the client.

The first four operations are write operations (destructive) and follow the same code path as for STORE (Fig. 1). Write operations are always transmitted over the TCP protocol to ensure retries in case of a communication error. STORE requests that exceed the server's memory capacity incur a cache eviction based on the least-recently-used (LRU) algorithm.

GET requests follow a similar code path (Fig. 2). If the key to be retrieved is actually stored in the table (a hit), the (key,value) pair is returned to the client. Otherwise (a miss), the server notifies the client of the missing key. One notable difference from the write path, however, is that clients can opt to use the faster but less-reliable UDP protocol for GET requests.

It is worth noting that GET operations can take multiple keys as an argument. In this case, Memcached returns all the KV pairs that were successfully retrieved from the table. The benefit of this approach is that it allows aggregating multiple GET requests in a single network packet, reducing network traffic and latencies. But to be effective, this feature requires that servers hold a large amount of RAM, so that servers are more likely to have multiple keys of interest in each request. (Another reason for large RAM per server is to amortize the RAM acquisition and operating costs over fewer servers.) Because some clients make extensive use of this feature, "wimpy" nodes are not a practical proposition for them, since they typically support smaller amounts of RAM per server.

\section{TILEPro64 Architecture}

Tile processors are a class of general-purpose and power-efficient many-core processors from Tilera using switched, on-chip mesh interconnects and coherent caches. The TILEPro64 is Tilera's second generation many-core processor chip, comprising 64 power efficient general-purpose cores connected by six $8 \mathrm{x} 8$ mesh networks. The mesh networks also connect the on-chip Ethernet, PCIe, 


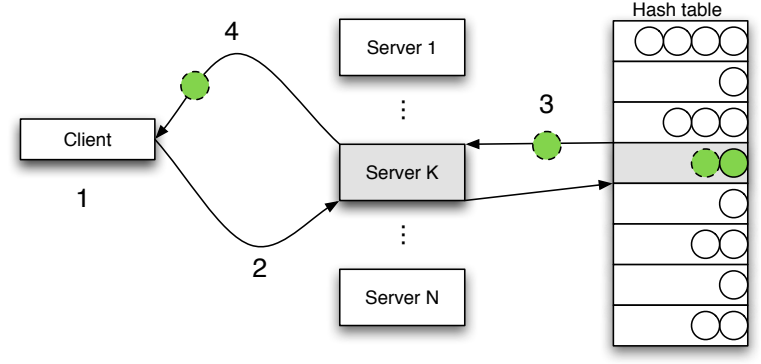

Figure 2: Read path: The client selects a server (1) by computing $k_{1}=$ consistent_hash $_{1}(k e y)$ and sends (2) it the key. The server calculates $k_{2}=h_{a s h}(k e y) \bmod M$ and looks up (3) a (key,value) pair in the appropriate slot $k_{2}$ in the hash table (and walks the chain of items if there were any collisions). Finally, the server returns (4) the (key, value) to the client or notifies it of the missing record.

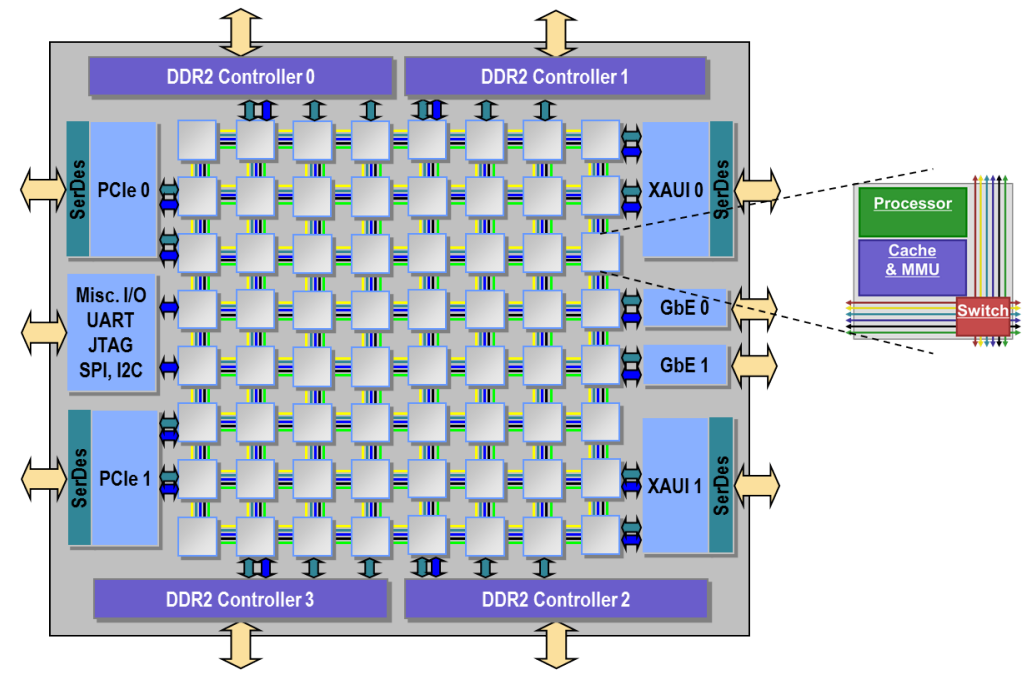

Figure 3: High-level overview of the Tilera TILEPro64 architecture. The processor is an 8x8 grid of cores. Each of the cores has a 3-wide VLIW CPU, a total of $88 \mathrm{~KB}$ of cache, MMU and six network switches, each a full 5 port 32 -bit-wide crossbar. I/O devices and memory controllers connect around the edge of the mesh network. 
and memory controllers. Cache coherence across the cores, the memory, and $\mathrm{I} / \mathrm{O}$ allows for standard shared memory programming. The six mesh networks efficiently move data between cores, I/O and memory over the shortest number of hops. Packets on the networks are dynamically routed based on a two-word header, analogous to the IP and port in network packets, except the networks are loss-less. Three of the networks are under hardware control and manage memory movement and cache coherence. The other three networks are allocated to software. One is used for $\mathrm{I} / \mathrm{O}$ and operating system control. The remaining two are available to applications in user space, allowing low-latency, low-overhead, direct communication between processing cores, using a user-level API to read and write register-mapped network registers.

Each processing core, shown as the small gray boxes in Fig. 3, comprises a 32-bit 5-stage VLIW pipeline with 64 registers, L1 instruction and data caches, L2 combined data and instruction cache, and switches for the six mesh networks. The $64 \mathrm{~KB}$ L2 caches from each of the cores form a distributed L3 cache accessible by any core and I/O device. The short pipeline depth reduces power and the penalty for a branch prediction miss to two cycles. Static branch prediction and in-order execution further reduce area and power required. Translation look-aside buffers support virtual memory and allow full memory protection. The chip can address up to 64GB of memory using four on-chip DDR2 memory controllers (greater than the 4GB addressable by a single Linux process). Each memory controller reorders memory read and write operations to the DIMMs to optimize memory utilization. Cache coherence is maintained by each cacheline having a "home" core. Upon a miss in its local L2 cache, a core needing that cache-line goes to the home core's L2 cache to read the cache-line into its local L2 cache. Two dedicated mesh networks manage the movements of data and coherence traffic in order to speed the cache coherence communication across the chip. To enable cache coherence, the home core also maintains a directory of cores sharing the cache line, removing the need for bus-snooping cache coherency protocols, which are power-hungry and do not scale to many cores. Because the L3 cache leverages the L2 cache at each core, it is extremely power efficient while providing additional cache resources. Figure 3 shows the I/O devices, 10G and 1GB Ethernet, and PCI-e, connecting to the edge of the mesh network. This allows direct writing of received packets into on-chip caches for processing and vice-versa for sending.

\section{Execution Model}

Although TILEPro64 has a different architecture and instruction set than the standard x86-based server, it provides a familiar software development environment with Linux, gcc, autotools, etc. Consequently, only a few software tweaks to basic architecture-specific functionality suffice to successfully compile and execute Memcached on a TILEPro64 system. However, this naïve port does not perform well and can hold relatively little data. The problem lies with Memcached's share-all multithreaded execution model (Fig. 4). In a standard 


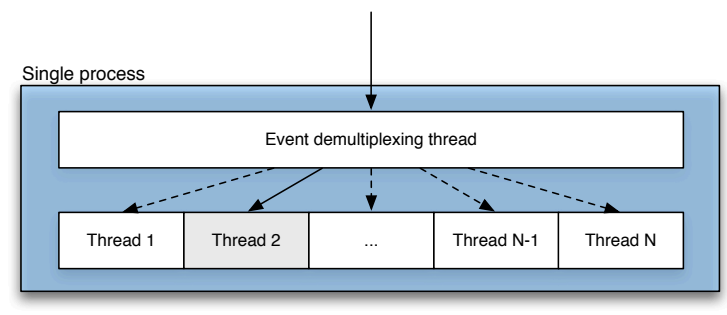

Figure 4: Execution model of standard version of Memcached.

version of Memcached, one thread acts as the event demultiplexer, monitoring network sockets for incoming traffic and dispatching event notifications to one of the $N$ other threads. These threads execute incoming requests and return the responses directly to the client. Synchronization and serialization are enforced with locks around key data structures, as well as a global hash table lock that serializes all accesses to the hash table. This serialization limits the scalability and benefits we can expect with many cores.

Moreover, recall that TILEPro64 has a 32-bit instruction set, limiting each process' address space to $2^{32}$ bytes (4GB) of data. As discussed in Sec. 2, packing larger amounts of data in a single node holds both a cost advantage (by reducing the overall number of servers) and a performance advantage (by batching multiple get requests together).

However, the physical memory limit on the TILEPro64 is currently 64GB, allowing different processes to address more than 4GB in aggregate. The larger physical address width suggests a solution to the problem of the 32-bit address space: extend the multithreading execution model with multiple independent processes, each having its own address space. Figure 5 shows the extended model with new processes and roles. First, two hypervisor cores handle I/O ingress and egress to the on-chip network interface, spreading I/O interrupts to the appropriate CPUs and generating DMA commands. The servicing of I/O interrupts and network layer processing (such as TCP/UDP) is now owned by $K$ dedicated cores, managed by the kernel and generating data for user sockets. These requests arrive to the main Memcached process as before, which contains a demultiplexing thread and $N$ worker threads. Note, however, that these worker threads are statically allocated to handle either TCP or UDP requests, and each thread is running on exactly one exclusive core. These threads do not contain KV data. Rather, they communicate with $M$ distinct processes, each containing a shard of the KV data table in its own dedicated address space, as follows:

When a worker thread receives a request, it identifies the process that owns the relevant table shard with modulo arithmetic. It then writes the pertinent request information in a small region of memory that is shared between the thread and the process, and had been previously allocated from the global pool using a memory space attribute. The worker thread then notifies the shard process of the request via a message over the on-chip user-level network to the 


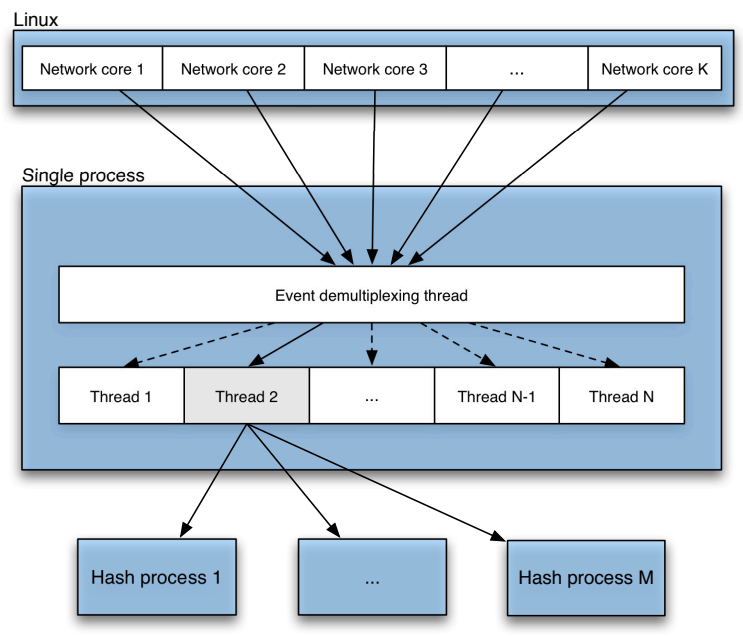

Figure 5: Execution model of Memcached on TILEPro64.

shard process (using a low-latency software interface). On a STORE request, the shard process copies the data into its private memory. For a GET operation, the shard process copies the requested value from its private hash table memory to the shared memory, to be returned to the requesting thread via the user-level network. For multi-GET operations, the thread merges all the values from the different shards into on the response packet.

This execution model solves the problem of the 32-bit address space limitations and that of a global table lock. Partitioning the table data allows each shard to comfortably reside within the 32-bit address space. Owning each table shard by a single process also means that all requests to mutate it are serialized and therefore require no locking protection. In a sense, this model adds data parallelism to what was purely task-parallel.

It is important to note that this execution model is much better suited for the TILEPro64 architecture than it would be for x86-based architectures. One reason is that communication between cores on x 86 goes through the memory hierarchy, which serializes and potentially slows down communication (especially if it ends up going through main memory). Another reason is that the TILEPro64 system-on-chip (SoC) offers higher flexibility for distributing network interrupts among an arbitrary number of cores. For the purposes of a fair comparison, we tuned our version of Memcached for the evaluated x86-systems using the existing execution model, to the best of our ability. 


\section{Experimental Evaluation and Discussion}

This section explores the performance of the modified Memcached on the TILEPro64 processor, and compares it to the tuned version on commodity x86-based servers.

\subsection{Methodology and Metrics}

We measure the performance of all servers by configuring them to run Memcached (only), using the following command line on x86:

memcached -p $11211-\mathrm{U} 11211$-u nobody -m <memory size>

and on the TILEPro64:

memcached -p 11211 -U 11211 -u root -m <memory size> -t \$tcp -V \$part

where \$tcp and \$part are variables representing how many TCP and hash partitions are requested (with the remaining number of cores allocated to UDP threads). On a separate client machine we use the mcblaster tool to stimulate the system under test and report the measured performance. A single run of mcblaster consists of two phases. During the initialization phase, mcblaster stores data in Memcached by sending requests at a fixed rate, the argument to $-\mathrm{W}$. This phase runs for 100 seconds (initialization requests are sent using the TCP protocol), storing 1,000,000 32-byte objects, followed by 5 seconds of idle time, with the command line:

mcblaster -z 32 -p 11211 -W 50000 -d 100 -k 1000000 -c 10 -r 10 <hostname>

During the subsequent phase, mcblaster sends query packets requesting randomly-ordered keys initialized in the previous phase and measures their response time using the command line:

mcblaster -z 32 -p 11211 -d 120 -k 1000000 -w 0 -c 20 -r \$rate <hostname>

where \$rate is a variable representing offered request rate.

We concentrate on two metrics of responsiveness and throughput. The first is the median response time (latency) of GET requests at a fixed offered load. The second, complementary, metric is the capacity of the system, defined as the approximate highest offered load (in transactions per second, or TPS) at which the mean response time remains under $1 \mathrm{~ms}$. Although this threshold is arbitrary, it is in the same order of magnitude of cluster-wide communications and well below the human perception level. We do not measure multi-GET requests because they exhibit the same read TPS performance as individual GET requests. Finally, we also measure the power usage of the various systems using Yokogawa WT210 power meter, measuring the wall power directly.

\subsection{Hardware Configuration}

The TILEPro64 S2Q system comprises a total of eight nodes, but we will focus our initial evaluation on a single node for a fairer comparison to the $\mathrm{x} 86$ 
nodes. In practice, all nodes have independent processors, memory, and networking, so the aggregate performance of multiple nodes scales linearly, and can be extrapolated from a single node's performance (we verified this assumption experimentally).

Our load-generating host contained a dual-socket quad-core Intel Xeon L5520 processor clocked at $2.27 \mathrm{GHz}$, with $72 \mathrm{~GB}$ of ECC DDR3 memory. It was also equipped with an Intel 82576 Gigabit ET Dual Port Server Adapter network interface controller that can handle transaction rates of over 500,000 packets/sec.

We used these systems in our evaluation:

- $1 \mathrm{U}$ server with single/dual socket quad-core Intel Xeon L5520 processor clocked at $2.27 \mathrm{GHz}(65 \mathrm{~W}$ TDP) and a varying number of ECC DDR3 8GB 1.35V DIMMs.

- $1 \mathrm{U}$ server single/dual socket octa-core AMD Opteron 6128 HE processor clocked at 2.0GHz (85W TDP) and a varying number of ECC DDR3 RAM DIMMs.

- Tilera $\mathrm{S}_{2} \mathrm{Q}^{5}$ : a $2 \mathrm{U}$ server built by Quanta Computer containing eight TILEPro64 processors clocked at $866 \mathrm{MHz}$, for a total of 512 cores. The system uses two power supplies (PSUs), each supporting two trays, which in turn each hold two TILEPro64 nodes. Each node holds 32GB of ECC DDR2 memory, a BMC, two GbE ports (we used one of these for this study), and two 10 Gigabit XAUI Ethernet ports.

We chose these low-power processors because they deliver a good compromise between performance and power compared to purely performance-oriented processors.

The Xeon server used the Intel $82576 \mathrm{GbE}$ network controller. We turned hyperthreading off since it empirically shows little performance benefit for Memcached, while incurring additional power cost. The Opteron server used the Intel $82580 \mathrm{GbE}$ controller. Both network controllers can handle packet rates well above our requirements for this study.

In most of our tests we used Memcached version 1.2.3i, running under CentOS Linux with kernel version 2.6.38.

\subsection{Experimental Setting}

\section{Core Allocation}

In our previous work [13] we focused mostly on exploring the significance of the static core allocation to roles. During our experiments, we observed that different core allocations among the 60 available cores (with 4 reserved for the Linux kernel), have substantial impact on performance. We systematically evaluated over 100 different allocations, testing for correlations and insights (including partial allocations that left some cores unallocated). From these experiments we have garnered the following observations:

\footnotetext{
${ }^{5}$ tilera.com/solutions/cloud_computing
} 
- The number of hash table processes determines the node's total table size, since each process owns an independent shard. But allocating cores beyond the memory requirements (in our case, 6 cores for a total of 24GB, leaving room for Linux and other processes), does not improve performance.

- The amount of networking cores does affect performance, but only up to a point. Above 12 networking cores, performance does not improve significantly, regardless of the number of UDP/TCP cores.

- TCP cores have little effect on UDP read performance, and do not contribute much after the initialization phase for read-only workloads. They do, however, affect TCP read and write capacity. This paper explores the significance of this choice further.

- Symmetrically, UDP cores play a role for UDP read capacity, so we have to balance the number of TCP and UDP cores to achieve satisfactory throughput meeting requirements for a given workload.

These experiments served to identify the highest-performance configurations for our evaluations. We thus fix the number of memory partitions to 6 and number of network cores to 8 . We vary the number of TCP and UDP cores such that they sum up to 46 cores. The remaining cores are reserved for the operating system and hardware drivers. We justify this allocation based on the observations that the number of hash partitions only determines the amount of accessible memory but does not affect the overall performance, and the sufficiency of 8 network cores for virtually all evaluated workloads.

\section{Aggregating Read and Write Throughput}

Typically, a production caching layer is used in read-dominated workloads, since that is its primary purpose. In this paper, however, we expand our evaluation to include more comprehensive workload compositions that include varying degrees of writes, and evaluate their effect on both performance and power consumption.

To evaluate these mixed workloads, we can no longer focus on read-only throughput as our performance metric. In this study, we define throughput as the aggregate of read and write transactions per second. We measured throughput while changing allocations of roles between worker cores. The configurations we used in the experiments are presented in table 1 . The 6 different mixes of operations were:

- $100 \%$ reads,

- $80 \%$ reads and $20 \%$ writes,

- $60 \%$ reads and $40 \%$ writes,

- $40 \%$ reads and $60 \%$ writes, 


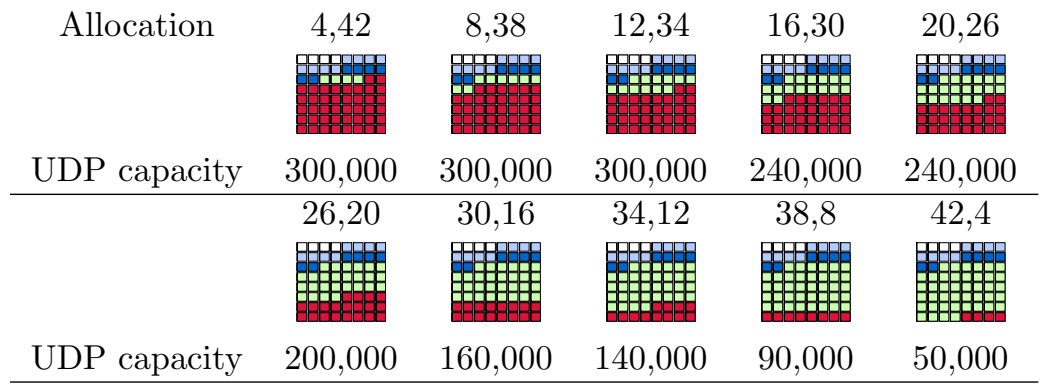

Figure 6: System capacity at various core allocations for a read-only workload. The numbers in each sequence represent the cores allocated to the TCP (green) and UDP (red) workers. The remaining cores are fixed and represent network cores (light blue) and hash table (dark blue). Linux always runs on 4 cores (white).

\begin{tabular}{lccccccccccc} 
Configurations & & & & & & & & & & \\
\hline TCP (cores) & 4 & 8 & 12 & 16 & 20 & 23 & 26 & 30 & 34 & 38 & 42 \\
\hline UDP (cores) & 42 & 38 & 34 & 30 & 26 & 23 & 20 & 16 & 12 & 8 & 4 \\
\hline
\end{tabular}

Table 1: Allocation of TCP and UDP roles between worker cores.

- $20 \%$ reads and $80 \%$ writes,

- $100 \%$ writes.

The data in the Appendix shows how the read and write throughput individually contribute to the overall throughput with difference core allocations.

\subsection{Effect of Workload Composition}

The best possible throughput for each configuration of cores is when both TCP and UDP cores are fully utilized. However, processing TCP traffic is much more involved than processing UDP traffic. The extra work stems from packetreception acknowledgment and re-transmissions associated with TCP, as well as protocol buffer and state management in the Linux kernel. This overhead renders TCP inherently slower than UDP. Under increasing traffic rate, this creates a situation wherein TCP cores achieve service latency of $1 \mathrm{~ms}$ much faster than UDP cores do. Per our defined methodology, we stop measuring throughput when either TCP or UDP response time crosses 1ms, ignoring the fact that the other cores are still capable of accepting more traffic. This behavior is demonstrated in Figure 8. We can clearly observe the TCP response latency reaching $1 \mathrm{~ms}$ while UDP response latency remains steady at approximately $210 \mu$ sec.

We can also see in Fig. 7 that adding more UDP cores has a positive effect on the aggregate throughput in read-only or read-heavy workloads, but as we start adding more write operations to the mix (these use TCP) the throughput drops steadily. This is a consequence of the TCP processing overhead where TCP 


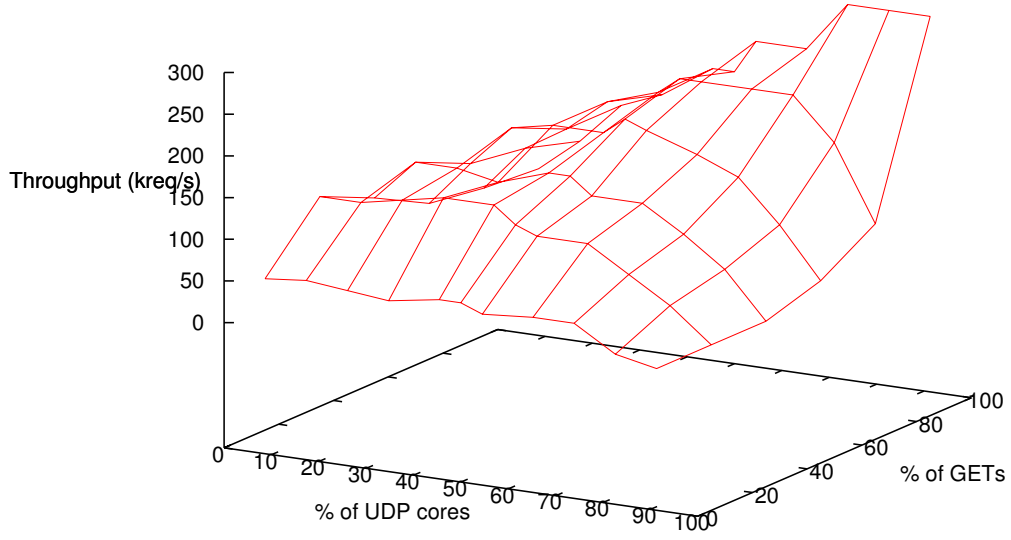

Figure 7: A surface describing the throughput of the TILEPro64 system based on the percentage of cores allocated to the UDP processing and the mix of STORE and GET operations.

cores saturate much faster, thus limiting the aggregate throughput. If the $1 \mathrm{~ms}$ requirement were dropped, the throughput would have been much higher, since UDP cores would still be able to accept more traffic in most configurations. Conversely, removing UDP cores and adding more TCP cores in their stead limits the read throughput but does not substantially increase the rate of write operations, effectively limiting the overall system throughput. This supports our previous finding that a balanced allocation of roles to cores is required for optimum performance on the TILEPro64.

The x86-based systems used the same Memcached execution model as before [13], but with some software improvements and tuning to reduce lock contention. In the $\mathrm{x} 86$ execution model, there is no need to allocate roles to cores, so we can measure performance in a single experiment. The throughput measurements for Opteron and Xeon systems are presented in Fig. 9. The clearly linear relationship between GET requests and read throughput. Similarly, write throughput grows roughly linearly with the proportion of STORE requests. It is interesting to note that while the rate of growth is dissimilar between the two servers and request types: the Xeon server appears to be better optimized for STORE requests than the Opteron, and in fact its write capacity even exceeds its own read throughput capacity. 


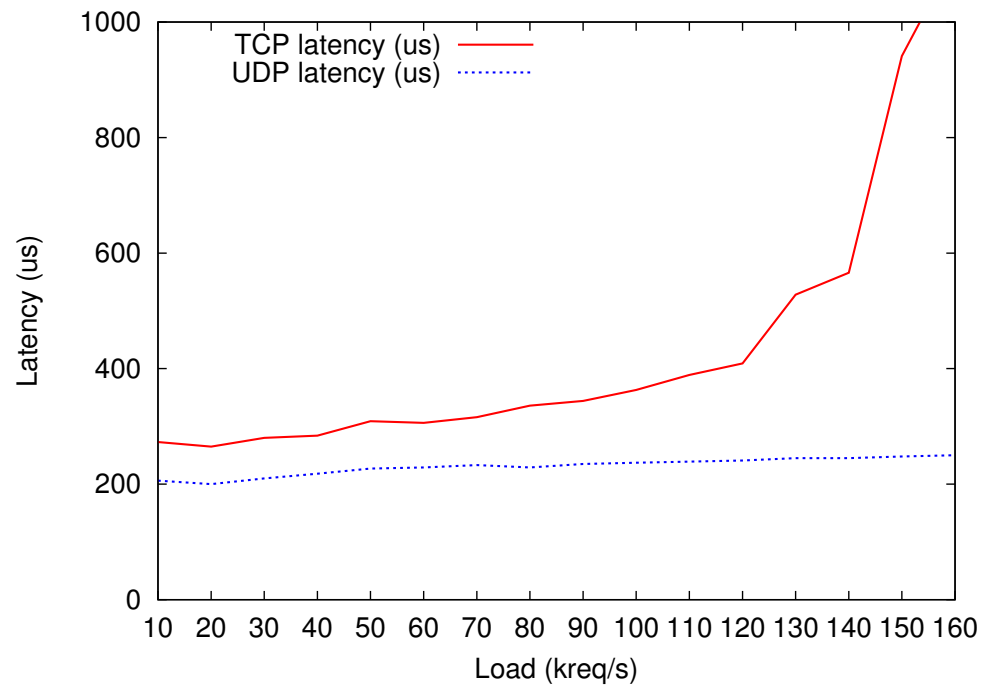

Figure 8: Latency of TCP and UDP responses for one of the tested core configurations on TILEPro64.

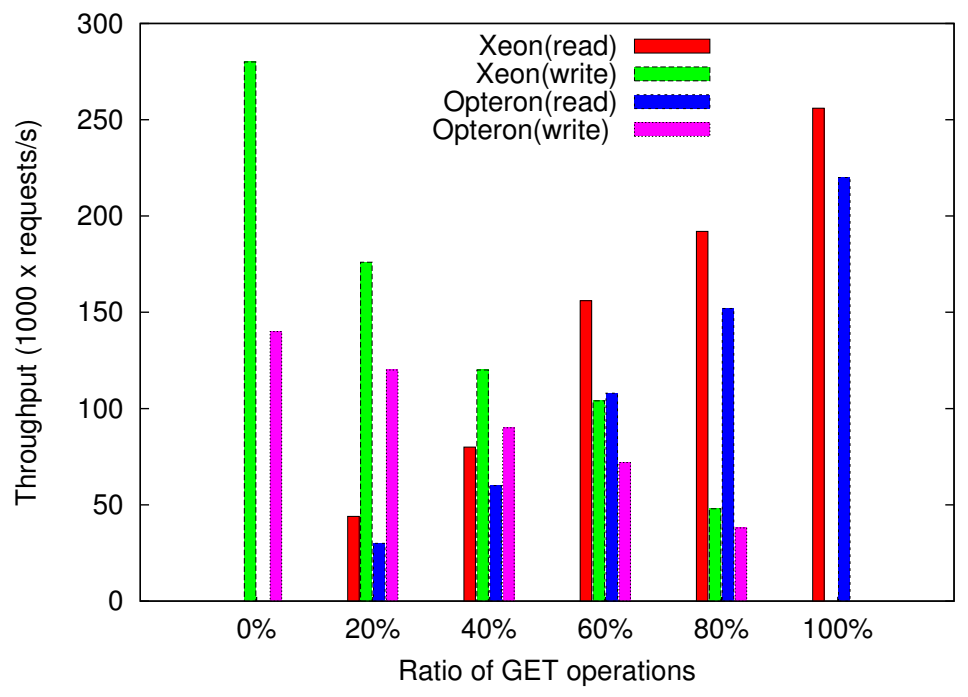

Figure 9: Throughput breakdown for Opteron and Xeon processors as the function of the percentage of read operations, using UDP for get requests. 


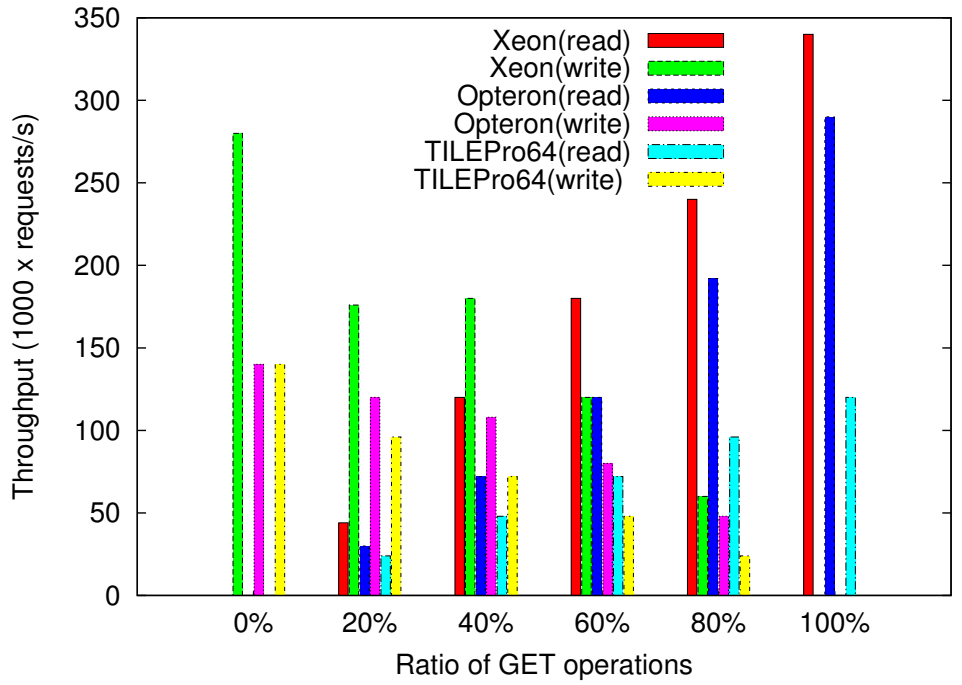

Figure 10: Throughput breakdown for Opteron, Xeon and TILEPro64 processors using TCP for both STORE and GET requests.

\subsection{Effect of Communication Protocol}

Another significant parameter is the transmission protocol for read requests. UDP is often used to trade reliability of TCP for lower latency in production environments. The performance differences are illustrated in in Fig. 8. We can see a latency advantage to UDP over TCP on the TILEPro64 (which occurs also on the $\mathrm{x} 86$-based systems). This advantage is readily explained by the fact that TCP is a transaction-based protocol and as such, it has a higher overhead associated with a large number of small packets. In fact, this overhead can grow so large that the faster cores on the x86-based systems can deliver higher throughput than the x86-based system in TCP-heavy workloads.

In this paper, we explore the effect of protocol on the performance by configuring all systems to use TCP only for both read and write operations. In TILEPro64's case this means assigning TCP role to all worker cores. The performance of this system is summarized and compared to x86 based systems in the Fig. 10. We can observe that when sending traffic over TCP only, both the Xeon and Opteron systems demonstrate higher capacity than the TILEPro64 system. This is a consequence of the TCP processing overhead as discussed above.

\subsection{Effect of Packet Size}

We also tested the effect of packet size (essentially value size) on read performance. Packet sizes are limited to the system's MTU when using the UDP protocol for Memcached, which in our system is 1,500 bytes. To test this param- 


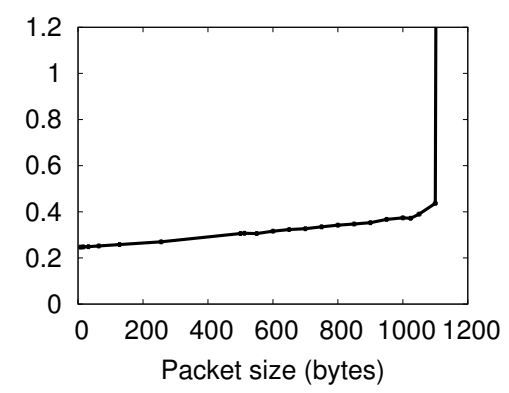

Figure 11: Median response time as a function of packet size.

eter, we fixed the read rate at $\lambda_{\text {size }}=100,000$ TPS and varied the payload size from 8 to 1,200 bytes. The results are presented in Fig. 11. The latency spike at the right is caused by the network's bandwidth limitation: sending 1,200-byte sized packets at the rate of $\lambda_{\text {size }}$ translates to a data rate of $960 \mathrm{Mbps}$, very close to the theoretical maximum of the $1 \mathrm{Gbps}$ channel. Because packet size hardly affects read performance across most of the range, and because we typically observe sizes of less than 100 bytes in real workloads, we set the packet size to 64 bytes in all experiments.

\subsection{Read Capacity Comparison Across Architectures}

Fig. 8 shows the median response time for GET requests under increasing load for TCP and UDP protocols, respectively. It is worth noting that UDP's lower latency often justifies its use over TCP, despite the latter's higher reliability and even throughput. Normal production traffic is typically below saturation throughput, so UDP's lower latencies are preferable to TCP.

We had previously exposed the difference between processors optimized for single-threaded performance vs. multi-threaded throughput [13]. The x86-based processors, with faster clock speeds and deeper pipelines, complete individual UDP GET requests $\approx 20 \%$ faster than the TILEPro64 across most load points. (Much of the response time is related to memory and network performance, where the differences are less pronounced.) This performance advantage is not qualitatively meaningful, because these latency levels are all under the $1 \mathrm{~ms}$ capacity threshold, providing adequate responsiveness. On the other hand, fewer cores, combined with centralized table and network management, translate to a lower saturation point and significantly reduced throughput for the x86-based servers when using a combination of TCP and UDP protocols. (When using TCP-only communication, session and state management overhead requires more powerful cores.)

This claim is corroborated when we analyze the scalability of Memcached as we vary the number of cores (Fig. 12 and 13). Here, the serialization in Memcached and the network stack prevents the x86-based architectures from scaling to even a few cores. The figure clearly shows that even within a single socket 


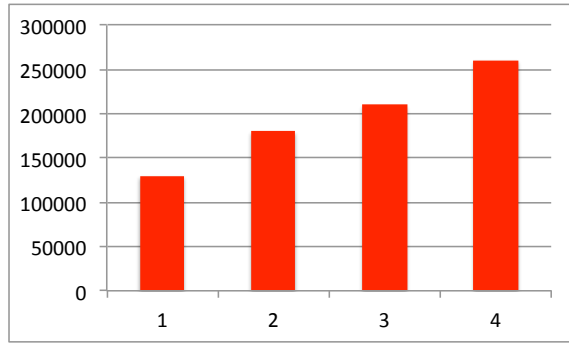

(a) Xeon

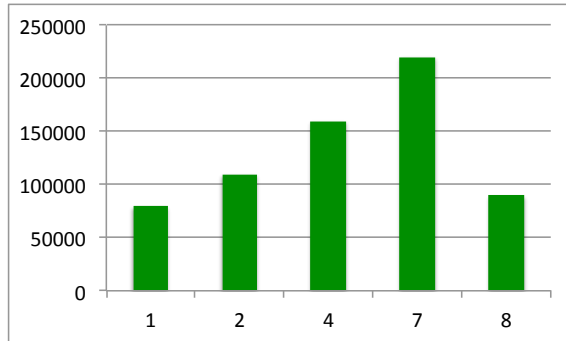

(b) Opteron

Figure 12: Scalability of Memcached on Xeon and Opteron systems with increasing number of cores. For x86, we simply change the number of Memcached threads with the -t parameter, since threads are pinned to individual cores.

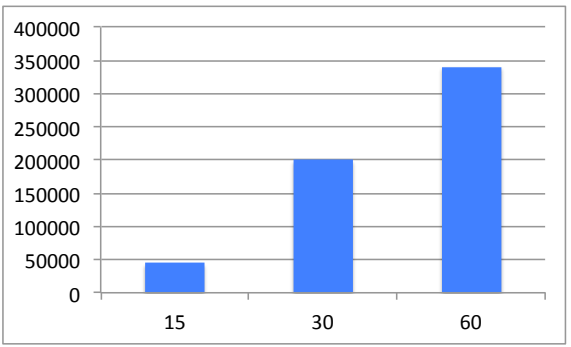

Figure 13: Scalability of TILEPro64 under the increasing number of cores. For TILEPro64 we turn off a number of cores and reallocate threads as in Fig. 6.

and with just 8 cores, performance scales poorly and cannot take advantage of additional threads. In fact, we must limit the thread count to 7 on the Opteron to maximize its performance. On the other hand, the TILEPro64 implementation can easily take advantage of (and actually requires) more cores for higher performance. Another aspect of this scaling shows in Fig. 6(e)-(f),(a), where UDP capacity roughly grows with the number of UDP cores. We do not know where this scaling would start to taper off, and will follow up with experiments on the 100-core TILE-Gx when it becomes available.

The sub-linear scaling on $\mathrm{x} 86$ suggests there is room for improvement in the Memcached software even for commodity servers. This is not a novel claim [15] and in fact we have managed to eek out much improved scalability compared to our previous effort [13]. But it drives the point that a different parallel architecture and execution model can scale much better.

\subsection{Power}

Table 2 shows the throughput and wall power drawn by each system while running at read capacity throughput. Node-for-node, the TILEPro64 delivers higher performance than the $\mathrm{x} 86$-based servers at comparable power. But the S2Q server also aggregates some components over several logical servers to save 


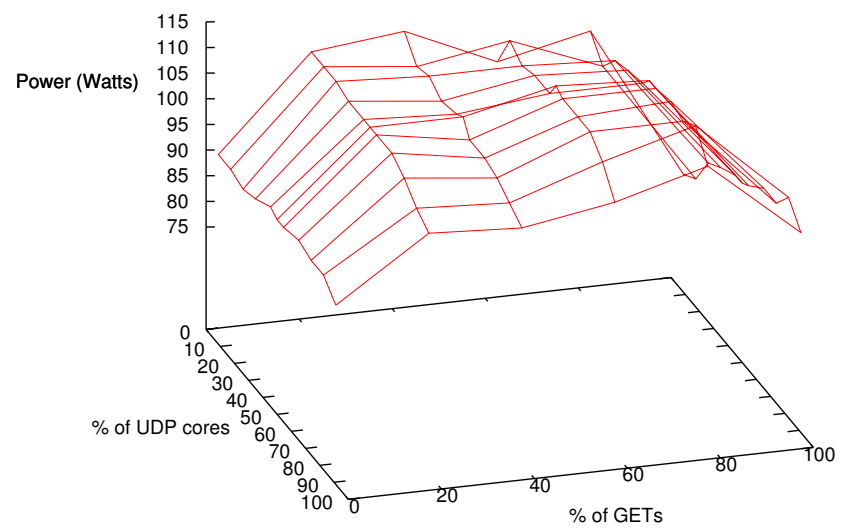

Figure 14: Power consumption of a TILEPro64 node as a function of the core configuration and workload mix.

power, such as: fans, BMC, and PSU. In a large data center environment with many Memcached servers, this feature can be very useful. Let us extrapolate these power and performance numbers to $256 \mathrm{~GB}$ worth of data, the maximum amount in a single S2Q appliance (extrapolating further involves mere multiplication).

As a comparison basis, we could populate the x86-based servers with many more DIMMs (up to a theoretical 384GB in the Opteron's case, or twice that if using 16GB DIMMs). But there are two operational limitations that render this choice impractical. First, the throughput requirement of the server grows with the amount of data and can easily exceed the processor or network interface capacity in a single commodity server. Second, placing this much data in a single server is risky: all servers fail eventually, and rebuilding the KV store for so much data, key by key, is prohibitively slow. So in practice, we rarely place much more than $64 \mathrm{~GB}$ of table data in a single failure domain. (In the S2Q case, CPUs, RAM, BMC, and NICs are independent at the 32GB level; motherboard are independent and hot-swappable at the 64GB level; and only the PSU is shared among $128 \mathrm{~GB}$ worth of data.)

Table 3 shows power and performance results for these configurations. Not only is the S2Q capable of higher throughput per node when using UDP than the $\mathrm{x} 86$-based servers, it also achieves it at lower power.

Another interesting observation is that power changes with workloads in case of TILEPro64. Figure 14 demonstrates this effect. For example, in readdominated workload, the power consumption decreases as we add more TCP cores. This is explained by the fact that TCP cores are used for writes only, and they are unused for read operations. With stock x86 Memcached this is 


\begin{tabular}{|c|c|c|c|}
\hline Configuration & RAM (GB) & Capacity (TPS) & Power (Watt) \\
\hline \hline $1 \times$ TILEPro64 (one node) & 32 & 335,000 & 94 \\
$2 \times$ TILEPro64 (one PCB) & 64 & 670,000 & 140 \\
$4 \times$ TILEPro64 (one PSU) & 128 & $1,340,000$ & 233 \\
\hline Single Opteron (UDP) & 64 & 220,000 & 109 \\
Single Opteron (TCP) & 64 & 290,000 & 121 \\
\hline Single Xeon (UDP) & 64 & 260,000 & 113 \\
Single Xeon (TCP) & 64 & 340,000 & 116 \\
\hline
\end{tabular}

Table 2: Power and capacity at different configurations. Performance differences at the singlesocket level likely stem from imbalanced memory channels. TILEPro64 measurements are done with mixed UDP/TCP configuration only - the latency and performance for the TCPonly configuration do not meet our operating requirements.

\begin{tabular}{|c|c|c|c|c|}
\hline Architecture & Nodes & Capacity & Power & TPS / Watt \\
\hline TILEPro64 & 8 (1 S2Q) & $2,680,000$ & 466 & 5,751 \\
Opteron & 4 & 880,000 & 436 & 2,018 \\
Xeon & 4 & $1,040,000$ & 440 & 2,363 \\
\hline
\end{tabular}

Table 3: Extrapolated power and capacity to 256GB.

not the case, as every core performs tasks both of TCP and UDP workers. In addition, switching to TCP-only mode of operation adds an extra 3 Watts of power in Xeon's case and 12 Watts in Opteron's case. We attribute this change in power consumption to the additional processing required for TCP traffic such as TCP session state management, packet receipt acknowledgments, buffer management and handling of fragmented packets; all of these are absent from UDP processing code. In addition, Opteron has double the number of cores than Xeon processor which further contributes to increase in power consumption. Another observation is that for either write-only or read-only workloads the power consumption is noticeably lower compared to mixed workloads. This is explained by the fact that TCP cores are unused when the machine serves UDP-only traffic and vice versa, UDP cores are unused when the machine serves TCP-only traffic.

\section{Scaling Power and Performance}

The TILEPro64 is limited by the total amount of memory per node, which means we would need more nodes than $\mathrm{x} 86$-based ones to fill large data requirements. To compare to a full S2Q box with $256 \mathrm{~GB}$, we can analyze a number of combinations of $\mathrm{x} 86$-based nodes that represent different performance and risk trade-offs. But if we are looking for the most efficient choice -in terms of throughput/Watt - then the best x86-based configurations in Table 2 have one socket with $64 \mathrm{~GB}$. Extrapolating these configurations to $256 \mathrm{~GB}$ yields the performance in Table 3.

Even compared to the most efficient Xeon configuration, the TILEPro shows 
a clear advantage in performance/Watt, and is still potentially twice as dense a solution in the rack ( $2 \mathrm{U}$ vs. $4 \mathrm{U}$ for $256 \mathrm{~GB})$.

\section{Conclusions and Future Work}

Low-power many-core processors are well suited to KV-store read-heavy workloads. Despite their low clock speeds, these architectures can perform on-par or better than comparably powered low-core-count x86 server processors. Our experiments show that a tuned version of Memcached on the 64-core Tilera TILEPro64 can yield at least $57 \%$ higher throughput than low-power x 86 servers at comparable latency. When taking power and node integration into account as well, a TILEPro64-based S2Q server with 8 processors handles at least two and a half times as many transactions per second per Watt as the x86-based servers with the same memory footprint. In addition, TILEPro64 cores enter very low power state when idle. Combined with role separation between cores, this leads to higher power efficiency. We have also found out that the choice of transport protocol can affect power consumption because of increased complexity. We have observed an increase of $\approx 3$ Watts on the Xeon and TILEPro64 and $\approx 12$ Watts on the Opteron when switching from UDP to TCP.

When taking power and node integration into account as well, a TILEPro64based S2Q server with 8 processors handles at least twice as many transactions per second per Watt as the $\mathrm{x} 86$-based servers with the same memory footprint. The main reasons for this advantage are the elimination or parallelization of serializing bottlenecks using the on-chip network; and the allocation of different cores to different functions such as kernel networking stack and application modules. This technique can be very useful across architectures, particularly as the number of cores increases.

In our study, the TILEPro64 exhibited near-linear throughput scaling with the number of cores, up to 46 UDP cores. One interesting direction for future research would be to reevaluate performance and scalability on the upcoming 64-bit 100-core TILE-Gx processor, which also supports 40 bits of physical address. Another interesting direction is to transfer the core techniques learned in this study to other KV stores, port them to TILEPro64 and measure their performance. Similarly, we could try to apply the same model to x 86 processors using multiple processes with their own table shard and no locks. But this would require a fast communication mechanism (bypassing main memory) that does not use global serialization such as memory barriers.

\section{Acknowledgments}

We would like to thank Ihab Bishara, Victor Li and Alvin Tang for their help and support of this paper. 
Appendix: Aggregate Performance on the TILEPro64 

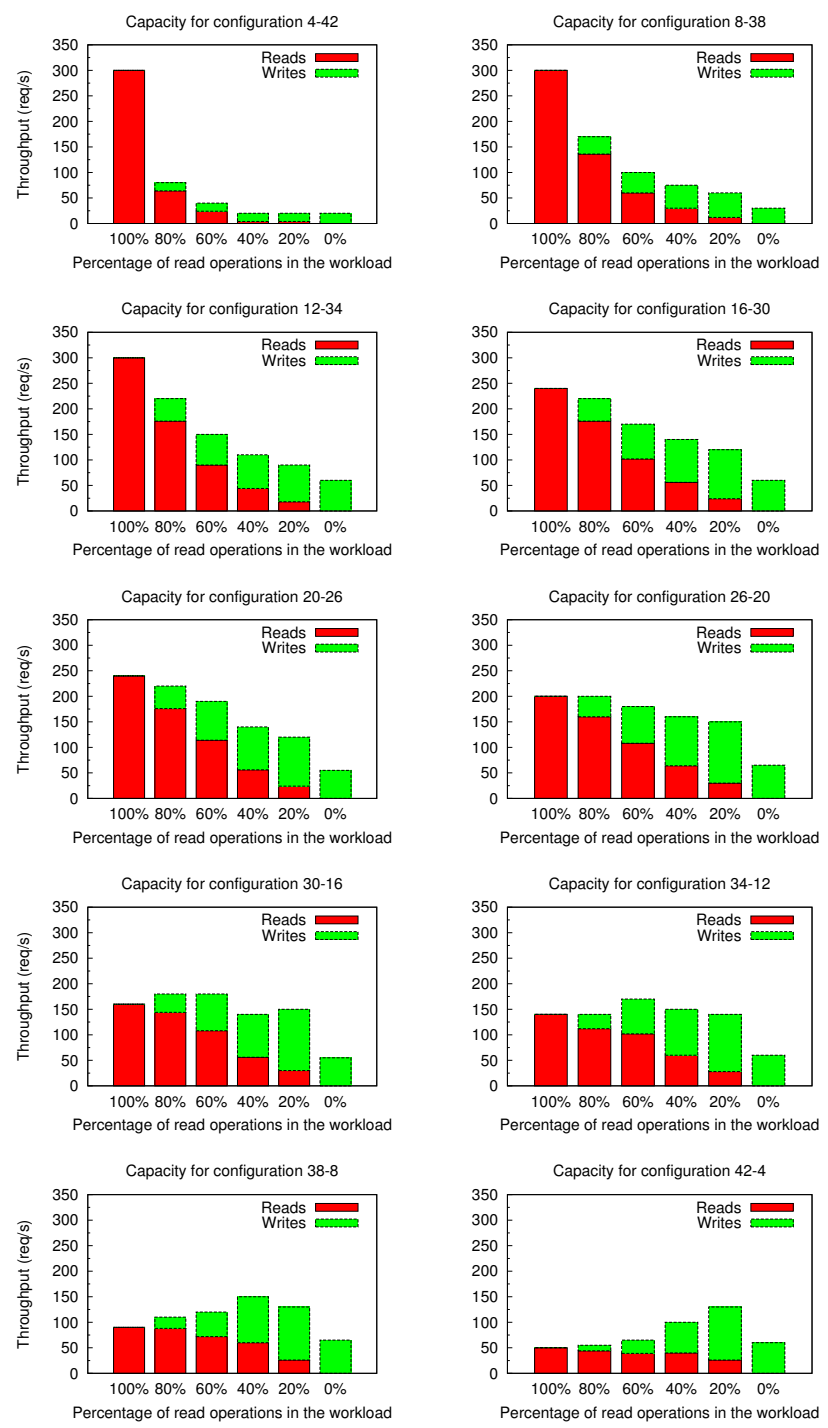

Figure 15: Throughput breakdown of different core allocations. The configuration names represent the split between UDP and TCP cores. The first number is number of UDP cores, the second one is the number of TCP cores. 


\section{References}

[1] G. DeCandia, D. Hastorun, M. Jampani, G. Kakulapati, A. Lakshman, A. Pilchin, S. Sivasubramanian, P. Vosshall, and W. Vogels, "Dynamo: Amazon's Highly Available Key-Value Store," in Proceedings of 21st ACM SIGOPS Symposium on Operating Systems Principles. New York, NY, USA: ACM, 2007, pp. 205-220.

[2] B. Fitzpatrick, "Distributed caching with Memcached," Linux Journal, vol. 2004, no. 124, p. 5, August 2004.

[3] J. Petrovic, "Using Memcached for Data Distribution in Industrial Environment," in Proceedings of the 3rd International Conference on Systems. Washington, DC, USA: IEEE Computer Society, 2008, pp. 368-372.

[4] D. G. Andersen, J. Franklin, M. Kaminsky, A. Phanishayee, L. Tan, and V. Vasudevan, "FAWN: A Fast Array of Wimpy Nodes," in Proceedings of the ACM SIGOPS 22nd Symposium on Operating Systems Principles. New York, NY, USA: ACM, 2009, pp. $1-14$.

[5] K. Lim, P. Ranganathan, C. Jichuan, P. Chandrakant, M. Trevor, and R. Steven, "Understanding and Designing New Server Architectures for Emerging WarehouseComputing Environments," Proceedings of 35th International Symposium on Computer Architecture, June 2008.

[6] W. Lang, J. M. Patel, and S. Shankar, "Wimpy Node Clusters: What About Non-wimpy Workloads?" in Proceedings of the 6th International Workshop on Data Management on New Hardware. New York, NY, USA: ACM, 2010, pp. 47-55.

[7] A. Greenberg, J. Hamilton, D. A. Maltz, and P. Patel, "The Cost of a Cloud: Research Problems in Data Center Networks," SIGCOMM Computuer Communication Review, vol. 39, no. 1, pp. 68-73, December 2008.

[8] B. Taylor, M., J. Kim, J. Miller, D. Wentzlaff, F. Ghodrat, B. Greenwald, H. Hoffman, J.-W. Lee, P. Johnson, W. Lee, A. Ma, A. Saraf, M. Seneski, N. Shnidman, V. Strumpen, M. Frank, S. Amarasinghe, and A. Agarwal, "The RAW Microprocessor: A Computational Fabric for Software Circuits and General Purpose Programs." IEEE Micro, April 2002 .

[9] B. Taylor, M., W. Lee, J. Miller, D. Wentzlaff, I. Bratt, B. Greenwald, H. Hoffmann, P. Johnson, J. Kim, J. Psota, A. Saraf, N. Shnidman, V. Strumpen, M. Frank, S. Amarasinghe, and A. Agarwal, "Evaluation of the RAW Microprocessor: An Exposed-WireDelay Architecture for ILP and Streams." Proceedings of the 31st International Symposium on Computer Architecture, June 2004.

[10] S. Bell, B. Edwards, and J. Amann, "Tile64-processor: A 64-core SoC with Mesh Interconnect," Solid-State Circuits, pp. 88-598, 2008.

[11] A. Agarwal, "The Tile Processor: A 64-core Multicore for Embedded Processing," Proceedings of 11th workshop on High Performance Embedded Computing, 2007.

[12] A. Agarwal, L. Bao, J. Brown, B. Edwards, M. Mattina, C.-C. Miao, C. Ramey, and D. Wentzlaff, "Tile Processor: Embedded Multicore for Networking and Multimedia." Proceedings of 19th Symposium on High Performance Chips, August 2007.

[13] M. Berezecki, E. Frachtenberg, M. Paleczny, and K. Steele, "Many-core Key-value Store," Proceedings of the 2nd International Green Computing Conference IGCC'11, July 2011. 
[14] D. Karger, A. Sherman, A. Berkheimer, B. Bogstad, R. Dhanidina, K. Iwamoto, B. Kim, L. Matkins, and Y. Yerushalmi, "Web Caching with Consistent Hashing," in Proceedings of the 8th International Conference on World Wide Web, New York, NY, USA, 1999, pp. 1203-1213.

[15] N. J. Gunther, S. Subramanyam, and S. Parvu, "A Methodology for Optimizing Multithreaded System Scalability on Multi-cores," CoRR, vol. abs/1105.4301, 2011. 